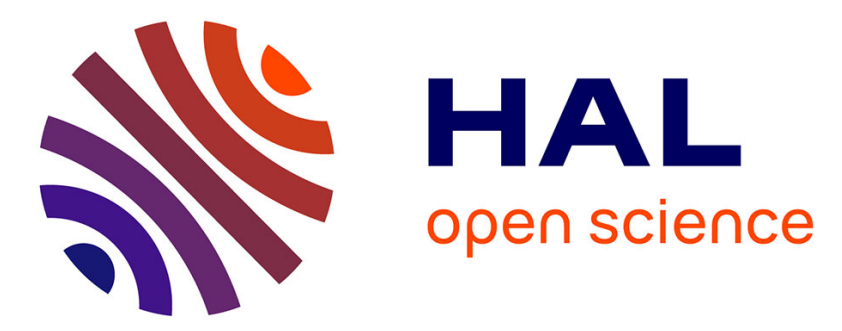

\title{
Functional brain imaging with M/EEG using structured sparsity in time-frequency dictionaries
}

Alexandre Gramfort, Daniel Strohmeier, Jens Haueisen, Matti Hamalainen, Matthieu Kowalski

\section{- To cite this version:}

Alexandre Gramfort, Daniel Strohmeier, Jens Haueisen, Matti Hamalainen, Matthieu Kowalski. Functional brain imaging with M/EEG using structured sparsity in time-frequency dictionaries. International Conference on Information Processing in Medical Imaging (IPMI '11), Jul 2011, Irsee, Germany. pp.600-611, 10.1007/978-3-642-22092-0_49 . inria-00605502

\section{HAL Id: inria-00605502 https://hal.inria.fr/inria-00605502}

Submitted on 1 Jul 2011

HAL is a multi-disciplinary open access archive for the deposit and dissemination of scientific research documents, whether they are published or not. The documents may come from teaching and research institutions in France or abroad, or from public or private research centers.
L'archive ouverte pluridisciplinaire HAL, est destinée au dépôt et à la diffusion de documents scientifiques de niveau recherche, publiés ou non, émanant des établissements d'enseignement et de recherche français ou étrangers, des laboratoires publics ou privés. 


\title{
Functional brain imaging with M/EEG using structured sparsity in time-frequency dictionaries
}

\author{
A. Gramfort ${ }^{123}$, D. Strohmeier ${ }^{4}$, J. Haueisen ${ }^{456}$, M. Hamalainen ${ }^{3}$, M. \\ Kowalski $^{7}$ \\ 1 INRIA, Parietal team, Saclay, France \\ 2 LNAO/NeuroSpin, CEA Saclay, Bat. 145, 91191 Gif-sur-Yvette Cedex, France \\ 3 Martinos Center, MGH Dept. of Radiology, Harvard Medical School, Boston, MA. \\ 4 Inst. of Biomedical Engineering and Informatics, Ilmenau University of Technology, \\ Ilmenau, Germany \\ ${ }^{5}$ Biomagnetic Center, Dept. of Neurology, University Hospital Jena, Jena, Germany \\ ${ }^{6}$ Dept. of Applied Medical Sciences, King Saud University, Riyadh, Saudi Arabia \\ 7 Laboratoire des Signaux et Systèmes (L2S), SUPELEC (C-4-20), Plateau de \\ Moulon, 91192 Gif-sur-Yvette Cedex, France
}

\begin{abstract}
Magnetoencephalography (MEG) and electroencephalography (EEG) allow functional brain imaging with high temporal resolution. While time-frequency analysis is often used in the field, it is not commonly employed in the context of the ill-posed inverse problem that maps the MEG and EEG measurements to the source space in the brain. In this work, we detail how convex structured sparsity can be exploited to achieve a principled and more accurate functional imaging approach. Importantly, time-frequency dictionaries can capture the non-stationary nature of brain signals and state-of-the-art convex optimization procedures based on proximal operators allow the derivation of a fast estimation algorithm. We compare the accuracy of our new method to recently proposed inverse solvers with help of simulations and analysis of real MEG data.
\end{abstract}

\section{Introduction}

Distributed source models in magnetoencephalography and electroencephalography (collectively M/EEG) use the individual anatomy derived from highresolution anatomical Magnetic Resonance Images (MRI). They employ a dense grid of current dipoles on the automatically segmented cortical surface. Following Maxwell's equations, each dipole adds its contribution linearly to the measured signal leading to a linear solution to the forward problem.

However, the number of sources by far exceeds the number of M/EEG sensors, making the inverse problem ill-posed. Therefore, constraints using a priori knowledge based on the properties of real sources are necessary. Common priors are based on the Frobenius norm. More recently, sparsity-inducing priors such 
as the $\ell_{1}$ norm have been introduced to take into account the assumption that only a few brain regions are typically active during a cognitive task.

While wavelet decompositions and time-frequency (TF) analysis are commonly computed from M/EEG data to exhibit transient oscillatory signals, the characteristics of such decompositions are rarely employed as a prior to regularize the inverse problem.

In this contribution, we propose to use both of these a priori assumptions within the framework of the inverse problem, making the $\mathrm{TF}$ analysis on the sensors optional. To do so, we propose to use a structured prior based on the $\ell_{21}$ mixed-norm combined with a simple $\ell_{1}$ norm. The prior is imposed on the coefficients of the TF decompositions using Gabor dictionaries.

Notation We indicate vectors with bold letters, a $\in \mathbb{R}^{N}$ (resp. $\mathbb{C}^{N}$ ) and matrices with capital bold letters, $\mathbf{A} \in \mathbb{R}^{N \times N}$ (resp. $\mathbb{C}^{N \times N}$ ). a $[i]$ stands for the $\mathrm{i}^{\text {th }}$ entry in the vector. We denote $\|\mathbf{A}\|_{\text {Fro }}$ the Frobenius norm, $\|\mathbf{A}\|_{\text {Fro }}^{2}=$ $\sum_{i, j=1}^{N}\left|\mathbf{A}_{i j}\right|^{2},\|\mathbf{A}\|_{1}=\sum_{i, j=1}^{N}\left|\mathbf{A}_{i j}\right|$ the $\ell_{1}$ norm, and $\|\mathbf{A}\|_{21}=\sum_{i=1}^{N} \sqrt{\sum_{j=1}^{N}\left|\mathbf{A}_{i j}\right|^{2}}$ the $\ell_{21}$ mixed norm. $\mathbf{A}^{T}$ and $\mathbf{A}^{\mathcal{H}}$ denote a matrix transpose and a Hermitian transpose, respectively.

The inverse problem with time-frequency dictionaries Given a linear forward operator $\mathbf{G} \in \mathbb{R}^{N \times P}$, also called lead field matrix or gain matrix, where $N$ is the number of sensors and $P$ the number of sources, the measurements $\mathbf{M} \in \mathbb{R}^{N \times T}$ ( $T$ number of time instants) are related to the source amplitudes $\mathbf{X} \in \mathbb{R}^{P \times T}$ by $\mathbf{M}=\mathbf{G X}$.

Solving the forward problem consists of computing $\mathbf{G}$ taking into account the electromagnetic properties of the head $[11,10]$, whereas in the inverse problem one computes a best estimate of the neural currents $\mathbf{X}^{\star}$ based on the measurements $\mathbf{M}$. However, to accomplish this task, priors need to be imposed on $\mathbf{X}$. The most conventional prior assumes that its weighted $\ell_{2}$ (Frobenius) norm is small. This corresponds to the family of Minimum-Norm (MN) inverse solvers [11, 3]. Several alternative solvers based on $\ell_{p}$ norms with $p<2$ have been also proposed. With $p \leq 1$, such priors promote sparse solutions [17,9]. Such priors however work on an instant by instant basis disregarding the oscillatory and nonstationary nature of electromagnetic brain signals. For this reason such solvers are usually employed following band-pass filtering of the data.

Beyond single instant solvers, various sparsity-promoting approaches have been proposed $[20,7,26]$. Although, they manage to capture the time courses of the activations, they implicitly assume that all active sources have non-zero activations throughout the analysis period. To go beyond this approach, we propose a solver where the sparsity of source configurations is promoted, but also where the time course of each active dipole is a linear combination of a few Gabor atoms. Our model can thus be expressed as:

$$
\mathbf{M}=\mathbf{G X}+\mathbf{E}=\mathbf{G Z} \Phi^{\mathcal{H}}+\mathbf{E},
$$

where $\boldsymbol{\Phi}^{\mathcal{H}} \in \mathbb{C}^{K \times T}$ is a dictionary of $K$ Gabor atoms, $\mathbf{Z} \in \mathbb{C}^{P \times K}$ are the coefficients of the decomposition, and $\mathbf{E}$ is additive white noise, $\mathbf{E} \sim \mathcal{N}(0, \lambda \mathbf{I})$. Note 

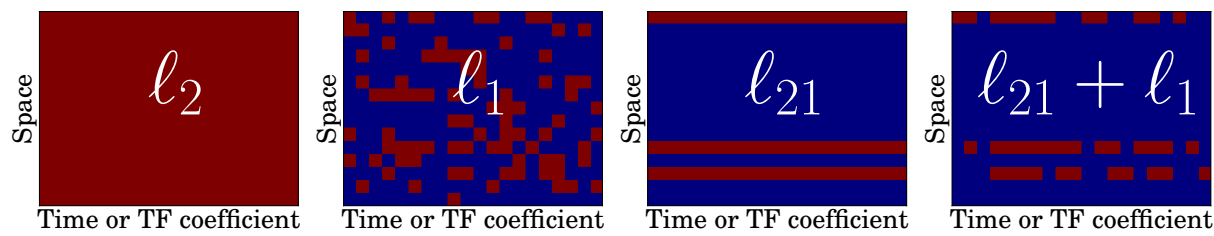

Fig. 1. Sparsity patterns promoted by the different priors $\left(\ell_{2}\right.$ no non-zero, $\ell_{1}$ scattered and unstructured non-zero, $\ell_{21}$ block row structure, $\ell_{21}+\ell_{1}$ block row structure with intra-row sparsity). Red indicates non-zero coefficients.

that this last assumption can be justified for M/EEG data as it is possible to estimate the noise covariance matrix from pre-stimulation recordings and spatially whiten the data. Given a prior on $\mathbf{Z}, \mathcal{P}(\mathbf{Z}) \sim \exp (-\Omega(\mathbf{Z}))$, the maximum a posteriori estimate (MAP) is obtained by solving:

$$
\mathbf{Z}^{\star}=\underset{\mathbf{Z}}{\arg \min } \frac{1}{2}\left\|\mathbf{M}-\mathbf{G} \mathbf{Z} \Phi^{\mathcal{H}}\right\|_{\text {Fro }}^{2}+\lambda \Omega(\mathbf{Z}), \lambda>0 .
$$

If we consider $\Omega(\mathbf{Z})=\|\mathbf{Z}\|_{1},(2)$ corresponds to a Lasso problem [23], also called Minimum Current Estimate (MCE) in the M/EEG literature [17], where features (or regressors) are spatio-temporal atoms. Similarly to the original formulation of MCE (i.e., with no $\mathbf{\Phi}$ ), such a prior is likely to suffer from inconsistencies over time [20]. Indeed such a norm does not impose a structure for the non-zero coefficients, that are likely to be scattered all over $\mathbf{Z}^{\star}$ (see Fig. 1). Therefore, simple $\ell_{1}$ priors do not guarantee that only a few sources are active during the time window of interest. To promote this, one needs to employ mixed-norms such as the $\ell_{21}$ norm [20]. By doing so, the estimates have a sparse row structure (see Fig. 1). However the $\ell_{21}$ prior on $\mathbf{Z}$ does not produce denoised time series as it does not promote source estimates that are formed by a sum of a few Gabor atoms. In order to recover the sparse row structure, while simultaneously promoting sparsity of the decompositions, we propose to use a composite prior formed by the sum of $\ell_{21}$ and $\ell_{1}$ norms. The prior then reads:

$$
\Omega(\mathbf{Z})=\rho\|\mathbf{Z}\|_{1}+(1-\rho)\|\mathbf{Z}\|_{21}, \quad 0<\rho<1 .
$$

Gabor dictionaries Here we briefly present some important properties of Gabor dictionaries (see [4] for more details). Given a signal observed over a time interval, its conventional Fourier transform estimates the frequency content but loses the time information. To analyze the evolution of the spectrum with time and hence the non-stationarity of the signal, Gabor introduced the windowed Fourier atoms which correspond to a short-time Fourier transform (STFT) with a gaussian window. In practice, for numerical computation, a challenge is to properly discretize the continuous STFT. The discrete version of the STFT is called the Gabor Transform. The setting we are considering is the finite dimensional one. Let $\mathbf{g} \in \mathbb{R}^{T}$ be a "mother" analysis window. Let $f_{0} \in \mathbb{N}$ and $k_{0} \in \mathbb{N}$ 
be the frequency and the time sampling rate of the time-frequency plane generated by the STFT, respectively. The family of the translations and modulations of the mother window generates a family of Gabor atoms $\left(\phi_{m f}\right)_{m f}$ forming the dictionary $\Phi \in \mathbb{C}^{T \times K}$. We denote the number of atoms by $K$. The atoms can be written

$$
\phi_{m f}[n]=\mathbf{g}\left[n-m k_{0}\right] e^{\frac{i 2 \pi f_{0} f n}{T}}, \quad m \in\left\{0, \ldots, \frac{T}{k_{0}}-1\right\}, f \in\left\{0, \ldots, \frac{T}{f_{0}}-1\right\} .
$$

If the product $f_{0} k_{0}$ is small enough, i.e., the time-frequency plane is sufficiently sampled, the family $\left(\phi_{m f}\right)_{m f}$ is a frame of $\mathbb{R}^{T}$, i.e., one can recover any signal $\mathbf{x} \in \mathbb{R}^{T}$ from its Gabor coefficients $\left(\left\langle\mathbf{x}, \phi_{m f}\right\rangle\right)=\boldsymbol{\Phi}^{\mathcal{H}} \mathbf{x}$. For the rest of the paper we assume that this condition is satisfied.

More precisely, there exists two constants $A, B>0$ such that

$$
A\|\mathbf{x}\|_{2}^{2} \leq \sum_{m, f}\left\langle\mathbf{x}, \phi_{m f}\right\rangle \leq B\|\mathbf{x}\|_{2}^{2} .
$$

When $A=B$, the frame is tight, and if $A=B=1$ then the frame is an orthogonal basis. The Balian-Low theorem says that it is impossible to construct a Gabor frame which is a basis. Consequently, a Gabor transform is redundant or overcomplete and there exists an infinitely number of ways to reconstruct $\mathbf{x}$ from a given family of Gabor atoms. In the following, $\boldsymbol{\Phi}$ is a frame.

The canonical reconstruction of $\mathbf{x}$ from its Gabor coefficients requires a canonical dual window, denoted by $\tilde{\mathbf{g}}$. Following (4) to define $\left(\tilde{\boldsymbol{\phi}}_{m f}\right)_{m f}$ we have:

$$
\mathbf{x}=\sum_{m, f}\left\langle\mathbf{x}, \phi_{m f}\right\rangle \tilde{\boldsymbol{\phi}}_{m f}=\sum_{m, f}\left\langle\mathbf{x}, \tilde{\boldsymbol{\phi}}_{m f}\right\rangle \phi_{m f}=\boldsymbol{\Phi}^{\mathcal{H}} \mathbf{x} \tilde{\boldsymbol{\Phi}}=\tilde{\boldsymbol{\Phi}}^{\mathcal{H}} \mathbf{x} \boldsymbol{\Phi}
$$

where $\tilde{\boldsymbol{\Phi}}$ is the Gabor dictionary formed with the dual windows. When the frame is tight, then we have $\tilde{\mathbf{g}}=\mathbf{g}$, and more particularly we have $\boldsymbol{\Phi} \boldsymbol{\Phi}^{\mathcal{H}}=\left\|\boldsymbol{\Phi} \boldsymbol{\Phi}^{\mathcal{H}}\right\| \mathbf{I}^{8}$. The representation being redundant, for any $\mathbf{x} \in \mathbb{R}^{T}$ one can find a set of coefficients $z_{m f}$ such that $\mathbf{x}=\sum_{m, f} z_{m f} \phi_{m f}$, while the $z_{m f}$ verify some suitable properties dictated by the application. For example, it is particularly interesting for M/EEG to find a sparse representation of the signal.

In practice, the Gabor coefficients are computed using the Fast Fourier Transform (FFT). The synthesis operation (and then, the inverse transform with the appropriate window) is accomplished with the inverse FFT and overlap-add techniques. Such analysis and synthesis operations are efficiently implemented in the LTFAT Matlab toolbox ${ }^{9}[22]$.

Related work Time-frequency analysis is commonly used in the context of M/EEG both in the sensor and source space, but rarely integrated with the solution of the inverse problem. Some earlier contributions, such as $[5,8,16]$, apply

\footnotetext{
${ }^{8}$ We can however say nothing about $\boldsymbol{\Phi}^{\mathcal{H}} \boldsymbol{\Phi}$ in general.

${ }^{9}$ http://ltfat.sourceforge.net/
} 
a 2-step approach. First TF atoms are estimated from sensor data, typically with greedy approaches like Matching Pursuit. Subsequently, the inverse problem is solved on the selected components using parametric [8], scanning [16] or distributed methods [5]. Such methods suffer from several limitations. They implicitly assume that the source waveforms correspond to single TF atoms, while real brain signals are better modeled by a combination of atoms. In addition, estimation errors made in the first step have a direct impact on the accuracy of the source estimates. This is a particularly critical issue since the first step does not take into account the biophysics of the problem, i.e., the solution of the forward problem.

Spatial sparsity of source configurations has also been a recurrent assumption to improve the resolution of the M/EEG inverse problem. Recently, priors based on the $\ell_{21}$ mixed-norm have been proposed to achieve rotation invariance [12] and to recover spatially sparse while temporally smooth solutions [20]. However, in [20], a part of the temporal smoothness is obtained by filtering the data and by using temporal basis functions obtained with an SVD. Alternatively, a sparsityinducing Bayesian formulation of the inverse problem has been proposed [7, 26]. However, these approaches make the strong assumption that the source time courses are stationary. For example, the estimation crucially depends on the time interval considered. Also the solutions obtained by these solvers are invariant with respect to the permutation of the columns of $\mathbf{M}$, i.e., the temporal sequence of the data is immaterial.

In [24], an inverse solver that models the transient and non-stationary responses in M/EEG is proposed. A probabilistic model with wavelet shrinkage is employed to promote spatially smooth time courses. The estimation however relies on model approximations with no guarantee on the solution obtained. The most related work to ours, beyond the field of M/EEG, is probably [18] where sparsity is also promoted on the TF decompositions. The related optimization problem, is however solved with a truncated Newton method which only applies to differentiable problems. The non-differentiability of the cost function is tackled by using smooth approximation in the minimization. Moreover, Newton methods are known to be fast in the neighborhood of the solution, but little is known about the global convergence rate. In [19], it is proved that a suitable Newton technique has the same rate of convergence as the accelerated first order schemes like one we are employing below.

In this contribution, we do not address the problem of learning spatial basis functions such as in $[25,2]$ as doing so makes the cost function non-convex which affects the speed of convergence of the algorithm and also makes the solvers dependent on the initialization.

\section{Optimization strategy}

The procedure we propose is based on first-order schemes that handle the optimization of any cost function $\mathcal{F}$ if it can be written as a sum of two terms: 1 smooth convex term $f_{1}$ with Lipschitz gradient and 1 convex term $f_{2}$, potentially 
non-differentiable [1]: $\mathcal{F}(\mathbf{Z})=f_{1}(\mathbf{Z})+f_{2}(\mathbf{Z})$. The cost function in (2) belongs to this category. However, we need to be able to compute the proximal operator associated to $f_{2}$.

Definition 1 (Proximity operator). Let $\varphi: \mathbb{R}^{M} \rightarrow \mathbb{R}$ be a proper convex function. The proximity operator associated to $\varphi$, denoted by $\operatorname{prox}_{\varphi}: \mathbb{R}^{M} \rightarrow \mathbb{R}^{M}$ reads:

$$
\operatorname{prox}_{\varphi}(\mathbf{Z})=\underset{\mathbf{V} \in \mathbb{R}^{M}}{\arg \min } \frac{1}{2}\|\mathbf{Z}-\mathbf{V}\|_{2}^{2}+\varphi(\mathbf{V}) .
$$

In the case of the composite prior in (3), the proximity operator is given by the following lemma.

Lemma 1 (Proximity operator for $\ell_{21}+\ell_{1}$ ). Let $\mathbf{Y} \in \mathbb{C}^{P \times K}$ be indexed by a double index $(p, k) . \mathbf{Z}=\operatorname{prox}_{\lambda\left(\rho\|\cdot\|_{1}+(1-\rho)\|\cdot\|_{21}\right)}(\mathbf{Y}) \in \mathbb{C}^{P \times K}$ is given for each coordinates $(p, k)$ by

$$
Z_{p, k}=\frac{Y_{p, k}}{\left|Y_{p, k}\right|}\left(\left|Y_{p, k}\right|-\lambda \rho\right)^{+}\left(1-\frac{\lambda(1-\rho)}{\sqrt{\sum_{k}\left(\left|Y_{p, k}\right|-\lambda \rho\right)^{+^{2}}}}\right)^{+}
$$

where for $x \in \mathbb{R},(x)^{+}=\max (x, 0)$, and by convention $\frac{0}{0}=0$.

This result is a corollary of the proximity operator derived for hierarchical group penalties recently proposed in [15]. The penalty described here can indeed be seen as a 2-level hierarchical structure, and the resulting proximity operator reduces to successively applying the $\ell_{21}$ proximity operator then the $\ell_{1}$ one.

The pseudo code is provided in Algorithm 1. The Lipschitz constant $\mathcal{L}$ of the gradient of the smooth term in (2) is given by the square of the spectral norm of the linear operator $\mathbf{Z} \rightarrow \mathbf{G} \mathbf{Z} \boldsymbol{\Phi}^{\mathcal{H}}$. We estimate it with the power iteration method.

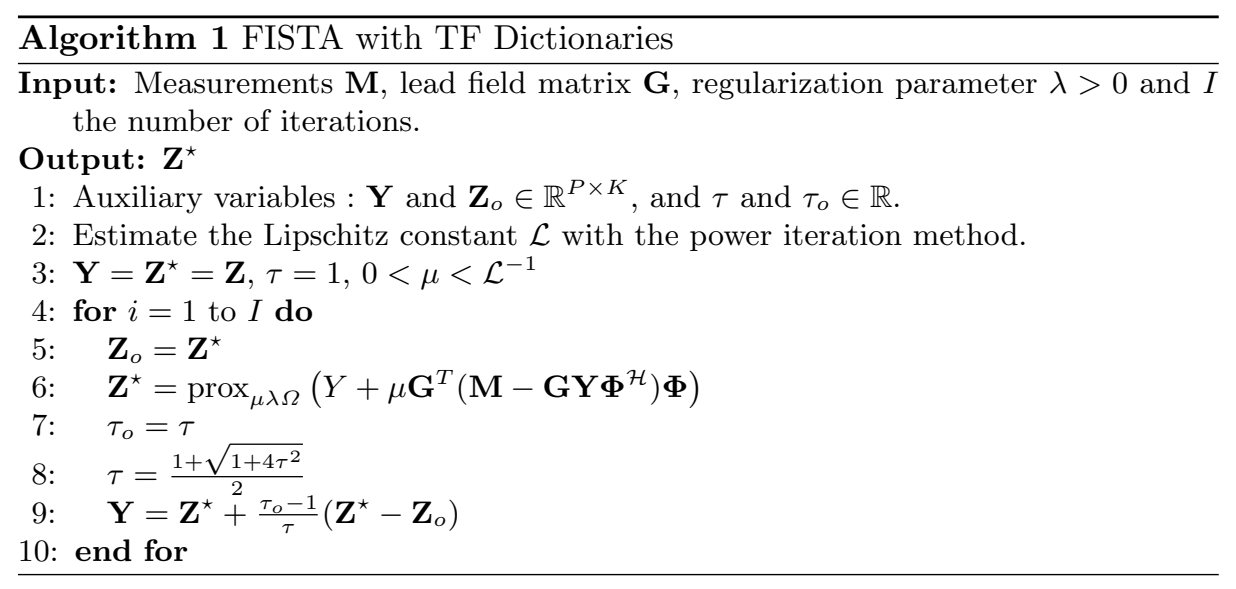


Source models with unconstrained orientations When the source orientations given by the normals of the cortical mesh cannot be trusted, it can be interesting to relax this constraint by placing three orthogonal sources at each spatial location. However, the TF composite prior also needs to be adapted. Assuming that each source is indexed by a spatial location $i$ and an orientation $o \in\{1,2,3\}$, the $\ell_{1}$ and $\ell_{21}$ norms read:

$$
\|\mathbf{Z}\|_{1}=\sum_{i k} \sqrt{\sum_{o=1}^{3}|\mathbf{Z}[(i, o), k]|^{2}} \quad \text { and } \quad\|\mathbf{Z}\|_{21}=\sum_{i} \sqrt{\sum_{k o}|\mathbf{Z}[(i, o), k]|^{2}}
$$

where $k$ indexes the TF coefficients. It amounts to grouping the orientations in a common $\ell_{2}$ norm such as in $[20,12]$.

Implementation Algorithm 1 requires to compute Gabor transforms at each iteration which can be computationally demanding. However, due to the $\ell_{21}$ sparsity inducing prior, only a few rows of $\mathbf{Z}$ have non-zeros coefficients. The Gabor transform is therefore computed for only a limited number of rows, equivalently a small number of active sources. This makes the computation of $\mathbf{Y} \Phi^{\mathcal{H}}$ (cf. Algorithm 1 line 6) much faster.

In order to significantly reduce the computation time of sparse regression problems, as the one presented here, a common strategy in machine learning is to use an active-set approach [21]. Intuitively, if one can verify the optimality of a solution (typically with Karush-Khun-Tucker (KKT) conditions), one can start by solving a small subproblem and then check if the solution obtained is optimal for the full problem. In the context of M/EEG, it consists in solving the inverse problem with a small set of sources, assuming the others have zero activation. This is particularly interesting when processing real M/EEG data for which $P$ can be up to 30000 , whereas only at most a few hundred sources are likely to be active. Whereas KKT optimality conditions can be efficiently verified with a $\ell_{21}$ penalty, it is not the case anymore with (3). To limit the computation time, we propose to address the problem in two steps. In a first step, the inverse problem is solved with a $\ell_{21}$ prior, using a small value for $\lambda$ and an active set strategy. Using a small $\lambda$ makes the active set larger than necessary, so that the active sources form a subset of it. Then (2) is solved with the composite prior on the restricted source space. By doing so, the computation on real data, such as those presented in Section 3.2, takes a few minutes on a standard laptop computer for given values of the regularization parameters.

Model selection Model selection amounts to setting the parameters $\lambda$ and $\rho$. As a principled way to do this, we use a k-fold cross-validation (CV) procedure in which the signal of sensors left out is predicted from the solution estimated using the remaining sensors. The best parameters are the ones that lead to the smallest average root mean square error (RMSE) between measurements and predictions across the folds. In practice, we use a 4 -fold CV with a logarithmic grid of 40 values for $\lambda$. To limit computation, we employed a fixed $\rho=0.1$, since our experiments proved it to be a good default choice. 
Fig. 2. Comparison of RMSE in the source space as a function of $\lambda$ $(\mathrm{SNR}=6 \mathrm{bB})$. Dashed lines correspond to results with no TF. TF priors improve the reconstruction and the best accuracy is obtained with the $\mathrm{TF} \ell_{21}+\ell_{1}$ prior. The 2-steps approach gives an almost optimal accuracy. The vertical dashed line

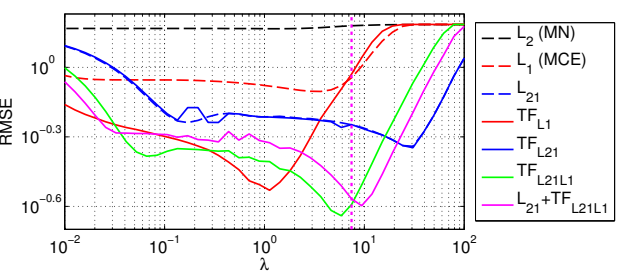
gives the $\lambda$ estimated by $\mathrm{CV}$.

\section{Results}

In the following, we first evaluate the accuracy of our solver on toy data and a simulated EEG dataset. We then apply our solver to experimental MEG data.

\subsection{Simulation study}

In order to have a reproducible and reasonably fast comparison of the different priors, we generated a small artificial dataset with 20 electrodes and 200 sources. 4 of these sources were randomly selected to be active. The ECD waveforms (cf. Fig. 3(a)) represent 1 high and 3 low frequency components. The time course of the oscillatory high frequency component is modeled by a Gabor atom, whereas the time courses of the low frequency components were obtained from a somatosensory evoked potential study [14] by fitting manually ECDs to the P15, N20 and P23 components. To make the comparison of the priors independent of the forward model and the sources spatial configuration, the linear forward operator was a random matrix, whose columns are normalized to 1 . White gaussian noise was added to the signals to achieve a desired signal-to-noise ratio (SNR). Following the notation of (1), we define SNR as $20 \log _{10}\left(\|M\|_{\text {Fro }} /\|E\|_{\text {Fro }}\right)$.

Figure 2 presents the RMSE on the estimation for different solvers as a function of $\lambda\left(\mathrm{RMSE}=\left\|\mathbf{X}_{\mathrm{sim}}-\mathbf{X}_{\Omega}^{\star}\right\|_{\text {Fro }}^{2}\right) . \lambda$ was chosen on a logarithmic grid from $10^{-2}$ to $10^{2}$ and $\rho$ was fixed to 0.1 . The Gabor dictionary is tight, constructed with a 128 samples long window $\mathrm{g}$ with $k_{0}=4$ samples time shift and $f_{0}=1$ sample frequency shift. Results show that the composite TF prior outperforms the other priors, while the 2-steps approach gives an almost optimal accuracy with a $\lambda$ estimated by CV. Figure 3 shows the reconstructions for the best $\lambda$ according to Fig. 2 for the $\ell_{1}, \ell_{21}$ and the TF composite priors. It can be observed, that the inverse method with the composite TF prior is able to reconstruct the smooth time course of the simulated sources contrary to $\ell_{1}$ and $\ell_{21}$ priors.

The TF composite prior was then challenged on a realistic EEG configuration with a 4-shell spherical head model (radii 0.90, 0.92, 0.96 and 1) and 60 electrodes placed according to the international 10-5 electrode system. The source waveforms were the same as before. The source space in Fig. 4 consisted of 152 sources in a regular grid (0.2 spacing) inside the innermost sphere. Source orientations were randomly selected. For depth compensation, a source covariance 


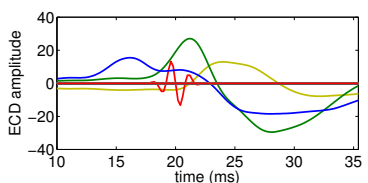

(a) $\mathbf{X}$ ground truth

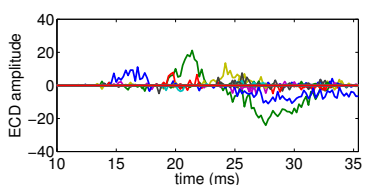

(d) $\mathbf{X}_{\ell_{1}}$

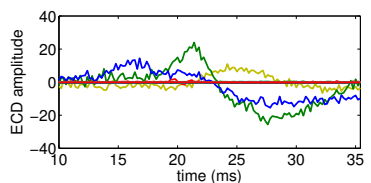

(g) $\mathbf{X}_{\ell_{21}}^{\star}$

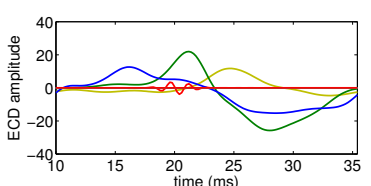

(j) $\mathbf{X}_{\mathrm{TF}}^{\star} \ell_{21}+\ell_{1}$

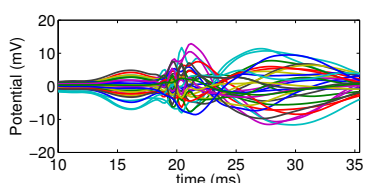

(b) $\mathbf{M}$ noiseless

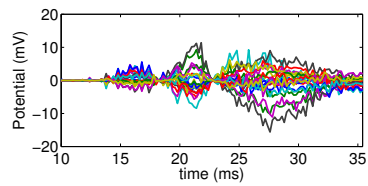

(e) $\mathbf{M}_{\ell_{1}}^{\star}=\mathbf{G X}_{\ell_{21}}^{\star}$

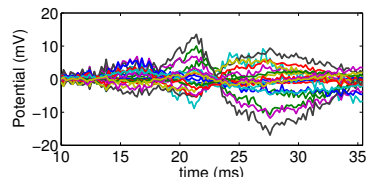

(h) $\mathbf{M}_{\ell_{21}}^{\star}=\mathbf{G} \mathbf{X}_{\ell_{21}}^{\star}$

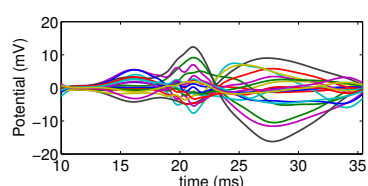

(k) $\mathbf{M}_{\mathrm{TF} \ell_{21}+\ell_{1}}^{\star}$

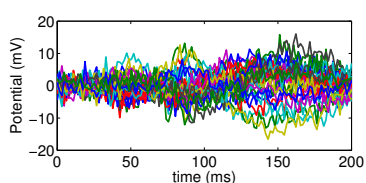

(c) $\mathbf{M}$ noisy $(\mathrm{SNR}=6 \mathrm{~dB})$

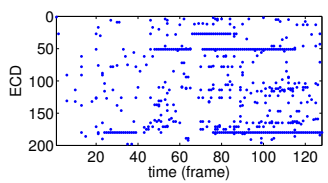

(f) Non-zeros of $\mathbf{X}_{\ell_{1}}^{\star}$

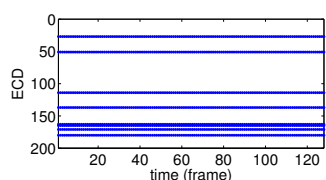

(i) Non-zeros of $\mathbf{X}_{\ell_{21}}^{\star}$

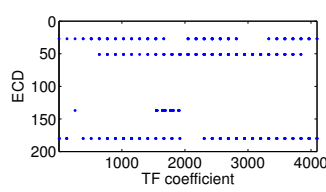

(l) Non-zeros of $\mathbf{Z}_{\ell_{21}+\ell_{1}}^{\star}$

Fig. 3. Simulations results with $S N R=6 d B$. (a) simulated source activations. (b) Noiseless simulated measurements. (c) Simulated measurements corrupted by noise. (de-f) Estimation with $\ell_{1}$ prior. (g-h-i) Estimation with $\ell_{21}$ prior [20]. (j-k-l) Estimation with composite TF prior. (f-i-l) show the sparsity patterns obtained by the 3 different priors as explained in Fig. 1. Result (j) shows how the composite TF prior improves over (d) and (g). (l) presents also a higher level of sparsity compared to (f) and (i).

based weighting method described in [13] was applied. Fig. 4 shows the head model and reconstructions obtained with $\ell_{21}$ and the $T F \ell_{21}+\ell_{1}$ priors. Even if the performance drops down due to the limited spatial resolution of EEG, the TF composite prior gives the best RMSE and is able to reconstruct and separate the high frequency component.

\subsection{Experimental results with MEG data}

We also applied our method to somatosensory MEG data. In this experiment, the right median-nerve was stimulated at the wrist with $0.2 \mathrm{~ms}$ constant current pulses above the motor threshold. The inter-stimulus interval was random between 3 - 12 s in an event-related design. MEG data were acquired using a 306-channel Neuromag Vectorview system. The signals were recorded with 
Fig. 4. Results with real EEG lead field $(\mathrm{SNR}=3 \mathrm{~dB})$. The 4 dipoles are color coded. Magenta dots show the $3 \mathrm{D}$ grid of sources. Dark dots show the EEG sensors locations. Contrary to $\mathrm{TF} \ell_{21}+\ell_{1}, \ell_{21}$ fails to recover the deep green dipole time course.
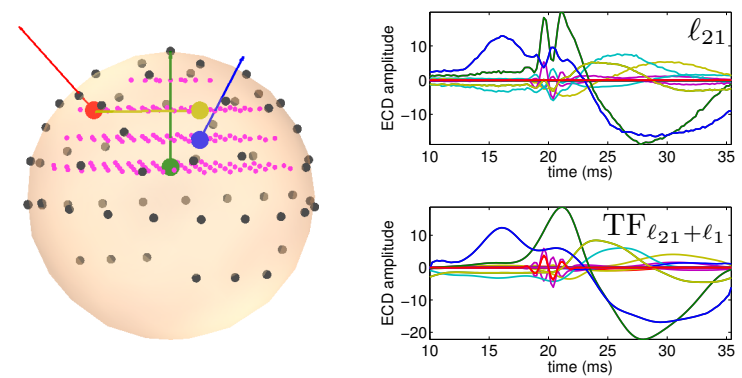

a bandpass of $0.01-250 \mathrm{~Hz}$, digitized at 1004 samples/s and averaged offline triggered by the stimulus onset. All epochs containing EOG signals higher than $150 \mu \mathrm{V}$ peak-to-peak amplitude were discarded from the averages, resulting in 68 averaged epochs. For source estimation, the noise-covariance matrix was estimated from the baseline period of $200 \mathrm{~ms}$ before stimulus onset in the raw data. The sources were estimated assuming unconstrained orientations. The Gabor dictionary is tight, constructed with a 256 samples $(\simeq 256 \mathrm{~ms})$ long window $\mathrm{g}$ with $k_{0}=16$ samples time shift and $f_{0}=1$ sample frequency shift. Results are presented in Fig. 5.

The first activation of the contralateral primary somatosensory cortex (cS1) peaks around $20 \mathrm{~ms}$ and lasts up to $100 \mathrm{~ms}$; then the secondary somatosensory cortices (contralateral cS2, ipsilateral iS2) activate around $70 \mathrm{~ms}$ and lasts up to $200 \mathrm{~ms}$. The posterior parietal cortex (PPC) starts to activate at $70 \mathrm{~ms}$ with a more significant activation between 140 and $200 \mathrm{~ms}$. This is consistent with the understanding of PPC, also known as the parietal association area, which is known to be higher in the hierarchy of cognitive processing [6].

\section{Conclusions}

In this work, we showed how physiologically motivated priors for brain activations can be accounted for in a mathematically principled framework in M/EEG source analysis. Using a composite prior, the sparsity of spatial patterns, the temporal smoothness, and the non-stationarity of the source signals were well recovered. Thanks to the structure of the cost function considered, mainly its convexity, an efficient optimization strategy was proposed. The problem being convex, the solver is not affected by improper initialization and cannot be trapped in local minima. Simulations indicated benefits of the approach over alternative solvers, while results with well understood MEG data confirm the accuracy of the reconstruction with real signals. Both results show that our solver is a promising new approach for mining M/EEG data.

Further work will investigate the impact of the choice of the time-frequency dictionary and the compromise between time and frequency resolution of the Gabor atoms, leading eventually to the use of a union of dictionaries. 
Fig. 5. Results obtained with the TF composite prior $\ell_{21}+\ell_{1}$ applied to somatosensory MEG data. Estimation was performed with unconstrained orientations on a set of 8195 cortical locations $\left(\mathbf{G} \in \mathbb{R}^{306 \times 24585}\right)$. Estimation leads to 37 active brain locations that have been clustered into 4 groups matching known functional regions. Clustering was done using k-means based on the source activation time courses. (e) illustrates the cascade of activation starting from cS1, to both $\mathrm{S} 2$ cortices and later $\mathrm{cPPC}$

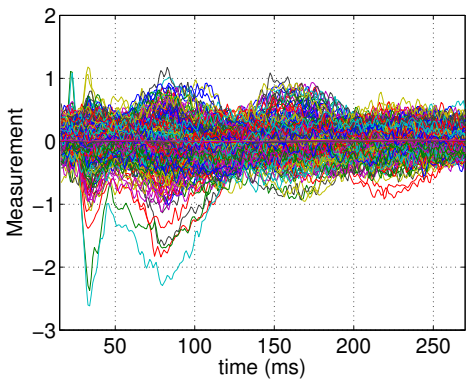

(a) MEG data: $\mathbf{M} \in \mathbb{R}^{306 \times 256}$

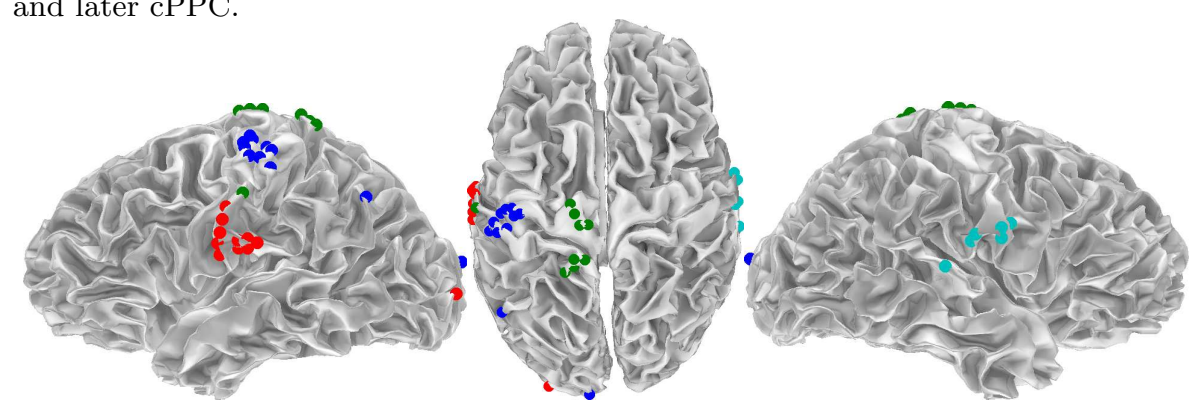

(b) Locations of the estimated active ECDs. ECDs clusters are color coded: Dark blue matches $\mathrm{cS} 1$, red cS2, light blue iS2, green cPPC.

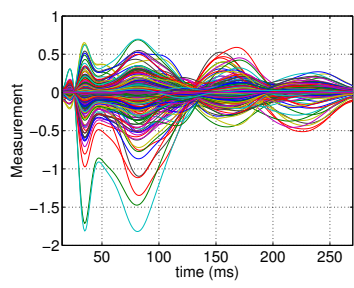

(c) $\mathbf{M}^{\star}=\mathbf{G X}^{\star}$ (denoised sensors data)

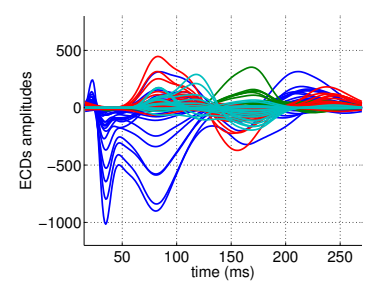

(d) $\mathbf{X}^{\star} \in \mathbb{R}^{24585 \times 256}$

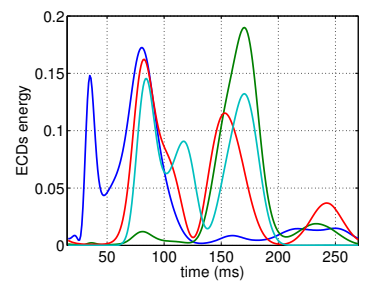

(e) Average energy density over time in each cluster.

\section{References}

1. Beck, A., Teboulle, M.: A fast iterative shrinkage-thresholding algorithm for linear inverse problems. SIAM Journal on Imaging Sciences 2(1), 183-202 (2009)

2. Bolstad, A., Veen, B.V., Nowak, R.: Space-time event sparse penalization for magneto-/electroencephalography. NeuroImage 46(4), 1066-81 (Jul 2009)

3. Dale, A., Liu, A., Fischl, B., Buckner, R.: Dynamic statistical parametric neurotechnique mapping: combining fMRI and MEG for high-resolution imaging of cortical activity. Neuron 26, 55-67 (2000)

4. Daubechies, I.: Ten lectures on Wavelets. SIAM-CBMS Conferences Series (1992)

5. Durka, P.J., Matysiak, A., Montes, E.M., Valdés-Sosa, P., Blinowska, K.J.: Multichannel matching pursuit and EEG inverse solutions. Journal of Neuroscience Methods 148(1), 49 - 59 (2005) 
6. Forss, N., Hari, R., Salmelin, R., Ahonen, A., Hamalainen, M., Kajola, M., Knuutila, J., Simola, J.: Activation of the human posterior parietal cortex by median nerve stimulation. Exp. Brain Res. (99), 309-315 (1994)

7. Friston, K., Harrison, L., Daunizeau, J., Kiebel, S., Phillips, C., Trujillo-Barreto, N., Henson, R., Flandin, G., Mattout, J.: Multiple sparse priors for the M/EEG inverse problem. Neuroimage 39(3), 1104-20 (Feb 2008)

8. Geva, A.B.: Spatio-temporal matching pursuit (SToMP) for multiple source estimation of evoked potentials. In: Electrical and Electronics Eng. pp. $113-116$ (1996)

9. Gorodnitsky, I., George, J., Rao, B.: Neuromagnetic source imaging with FOCUSS: a recursive weighted minimum norm algorithm. Electroencephalography and clinical Neurophysiology (Jan 1995)

10. Gramfort, A., Papadopoulo, T., Olivi, E., Clerc, M.: OpenMEEG: opensource software for quasistatic bioelectromagnetics. BioMed Eng OnLine 9(1), 45 (2010)

11. Hämäläinen, M., Ilmoniemi, R.: Interpreting magnetic fields of the brain: minimum norm estimates. Med Biol Eng Comput 32(1), 35-42 (Jan 1994)

12. Haufe, S., Nikulin, V.V., Ziehe, A., Müller, K.R., Nolte, G.: Combining sparsity and rotational invariance in EEG/MEG source reconstruction. NeuroImage 42(2), 726-38 (Aug 2008)

13. Haufe, S., Tomioka, R., Dickhaus, T., Sannelli, C., Blankertz, B., Nolte, G., Müller, K.R.: Large-scale EEG/MEG source localization with spatial flexibility. NeuroImage 54(2), 851-859 (2011)

14. Jaros, U., Hilgenfeld, B., Lau, S., Curio, G., Haueisen, J.: Nonlinear interactions of high-frequency oscillations in the human somatosensory system. Clin Neurophysiol 119(11), 2647-57 (2008)

15. Jenatton, R., Mairal, J., Obozinski, G., Bach, F.: Proximal methods for hierarchical sparse coding. In: ICML (2010)

16. Lelic, D., Gratkowski, M., Valeriani, M., Arendt-Nielsen, L., Drewes, A.M.: Inverse modeling on decomposed electroencephalographic data: A way forward? Journal of Clinical Neurophysiology 26(4), 227-235 (2009)

17. Matsuura, K., Okabe, Y.: Selective minimum-norm solution of the biomagnetic inverse problem. IEEE Trans Biomed Eng 42(6), 608-615 (June 1995)

18. Model, D., Zibulevsky, M.: Signal reconstruction in sensor arrays using sparse representations. Signal Processing 86(3), 624 - 638 (2006)

19. Nesterov, Y., Polyak, B.: Cubic regularization of newton's method and its global performance. Mathematical Programming 108(1), 177-205 (2006)

20. Ou, W., Hämaläinen, M., Golland, P.: A distributed spatio-temporal EEG/MEG inverse solver. NeuroImage 44(3), 932-946 (Feb 2009)

21. Roth, V., Fischer, B.: The group-lasso for generalized linear models: uniqueness of solutions and efficient algorithms. In: ICML. pp. 848-855 (2008)

22. Soendergard, P., Torrésani, B., Balazs, P.: The linear time frequency toolbox. Tech. rep., Technical University of Denmark (2009)

23. Tibshirani, R.: Regression shrinkage and selection via the lasso. Journal of the Royal Statistical Society Serie B 58(1), 267-288 (1996)

24. Trujillo-Barreto, N.J., Aubert-Vázquez, E., Penny, W.D.: Bayesian M/EEG source reconstruction with spatio-temporal priors. Neuroimage 39(1), 318-35 (2008)

25. Valdés-Sosa, P.A., Vega-Hernández, M., Sánchez-Bornot, J.M., Martínez-Montes, E., Bobes, M.A.: EEG source imaging with spatio-temporal tomographic nonnegative independent component analysis. HBM 30(6), 1898-910 (Jun 2009)

26. Wipf, D., Nagarajan, S.: A unified Bayesian framework for MEG/EEG source imaging. Neuroimage 44(3), 947-966 (Feb 2009) 